\title{
Ultrafast Magneto-Optical and Magnetization-Induced Second Harmonic Generation Techniques for Studies of Magnetic Nanostructures
}

\author{
A. BONDA*, S. UBA AND L. UBA \\ Institute of Computer Science, University of Białystok, Lipowa 41, PL-15-424 Białystok, Poland \\ The work concerns application of titanium-sapphire femtosecond laser oscillator with $82 \mathrm{MHz}$ repetition \\ rate at $800 \mathrm{~nm}$ in measurements of ultrafast magneto-optical and magnetization-induced second harmonic \\ generation effects. Time-resolved magneto-optical Kerr effect pump-probe method was used in investigation \\ of ultrafast precession of magnetization in $\mathrm{Fe} / \mathrm{Au}$ multilayers. Magnetization precession frequency, obtained \\ as a function of magnetic field applied, allows determining saturation magnetization and damping factor. The \\ magnetization-induced second harmonic generation effect has been used for study of garnet films. This method \\ allows to determine crystallographic symmetry and hysteresis loops in an external magnetic field at $2 \omega$ laser \\ induced frequency. The simultaneous measurements of linear and second order magneto-optical effects enable to \\ investigate magnetic interfaces in the studied structures.
}

PACS: 06.60.Jn, 78.47.J-, 42.65.Re, 75.50.-y

\section{Introduction}

Ultrafast techniques based on femtosecond laser pulses are being intensively developed for over a decade and applied in studies of dynamics and nonlinear effects in magnetic nanostructures [1]. It has been shown firstly on ferromagnetic Ni film that the ultrafast manipulation of spin state in subpico- and femtosecond time scale is possible by optical femtosecond laser pulses. Recent studies of laser-induced phenomena in various magnetic materials give promise in future for application in femtooptical devices, where single laser pulse will control logical nanobits. In this paper we present application of femtosecond laser oscillator in studying two effects induced by ultrafast laser pulses in magnetic materials. The first one is the ultrafast precession of magnetization vector measured with time-resolved pump-probe magneto-optical Kerr effect (TRMOKE) [2] method on $\mathrm{Fe} / \mathrm{Au}$ multilayers. The second is nonlinear phenomenon of magnetization-induced second harmonic generation (MSHG) [1] studied in garnet films.

\section{Experimental setup}

A schematic sketch of experimental setup is shown in Fig. 1. On the base of commercial laser system from Spectra-Physics which includes Ti:sapphire Tsunami femtosecond oscillator and $\mathrm{Nd}: \mathrm{YVO}_{4}$ Millennia laser capable of stable $5 \mathrm{~W}$ pumping at wavelength of $\lambda=$ $532 \mathrm{~nm}$, two experimental setups were arranged (Fig. 1). One setup is configured to measure TRMOKE and other is for MSHG, with simultaneous linear magneto-optical

\footnotetext{
* corresponding author; e-mail: a.bonda@uwb.edu.pl
}

Kerr effect measurements as an additional option. The oscillator generates about $\approx 400 \mathrm{~mW}$ of average power at repetition rate $82 \mathrm{MHz}$ and wavelength $\lambda \approx 800 \mathrm{~nm}$. Spectral parameters of the Tsunami: central wavelength and bandwidth were monitored at the output of the laser with a spectrometer (SPM) and adjusted depending on the requirements of individual experiments. Initial bandwidth operation was $\approx 90 \mathrm{~nm}$, and pulse width $\approx 30 \mathrm{fs}$. For detection of rapid process dynamics a stroboscopic pump-probe method was applied. Figure la shows a schematic sketch of the experimental setup. An important element of the setup is the optical delay line (ODL) with mounted on it retro-reflector $(\mathrm{R})$, reflecting back incident laser light. The task of ODL is to introduce variable delay time $\Delta \tau$ between pump light pulse which is used to excite the sample and weak probe beam pulse to measure changes in the optical properties. The $\Delta \tau$ is proportional to the difference in optical paths between pump and probe beams. The laser beam is directed onto the ODL via an optical beam splitter (BS), which reflects approximately $5 \%$ of the light creating a probe beam. Rest of the light forms a high intensity pumping beam. The sensitivity of the measurements was increased by applying double modulation method [3]. The modulation of the probe beam done by photoelastic modulator (PEM) at $f=50 \mathrm{kHz}$ was accompanied by modulation of pump beam with mechanical chopper (C) at $f=1.03 \mathrm{kHz}$. To visually control focused spots each of the beam independently, and also fix their spatial overlapping, CCD camera was used. Estimated size of the spots are $20 \mu \mathrm{m}$ and $30 \mu \mathrm{m}$ for probe and pump beam, respectively.

Zero difference in optical path length of both overlapping spots beams defines the zero scale position of the ODL, i.e., beginning of scale of $\Delta \tau$. In Fig. 2c result of time-resolved reflectivity $\Delta R / R$ measured with sin- 


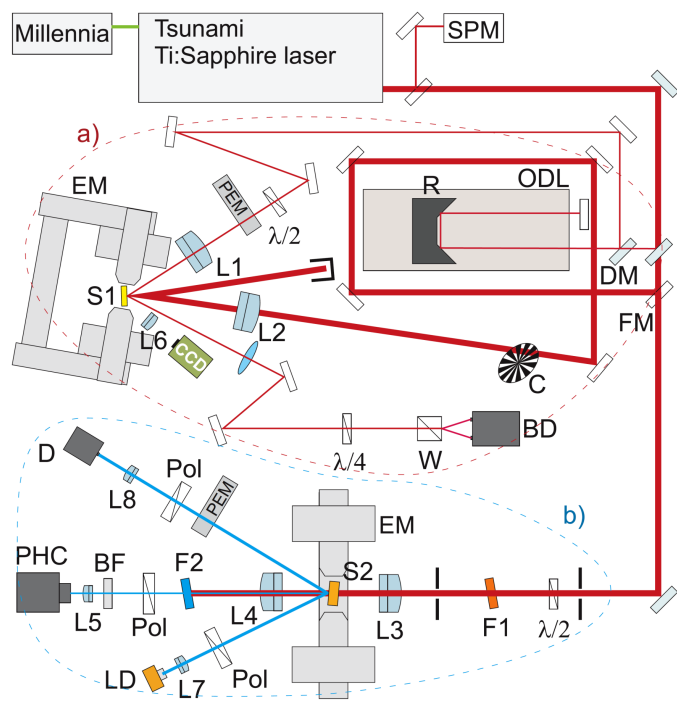

Fig. 1. A schematic sketch of experimental setups. (a) TRMOKE setup: FM - flipping mirror, DM dichroic mirror, ODL - optical delay line, $\mathrm{R}$ - retro-reflector, $\lambda / 2$ - halfwave plate, PEM - photoelastic modulator, $\mathrm{C}$ - beam chopper, $\mathrm{S} 1$ - sample, $\lambda / 4$ - quarterwave plate, $\mathrm{W}$ - Wollaston polarizer, BD - balanced photodetector. (b) MSHG setup: $\lambda / 2$ - halfwave plate, F1 - OG57 filter, S2 - sample, F2 - BG39 filter, Pol - polarizer/analyzer, BF bandpass filter, D - photodiode detector, LD - laser diode, PHC - photon counter. L1-L8 - achromatic objectives, EM - electromagnets.

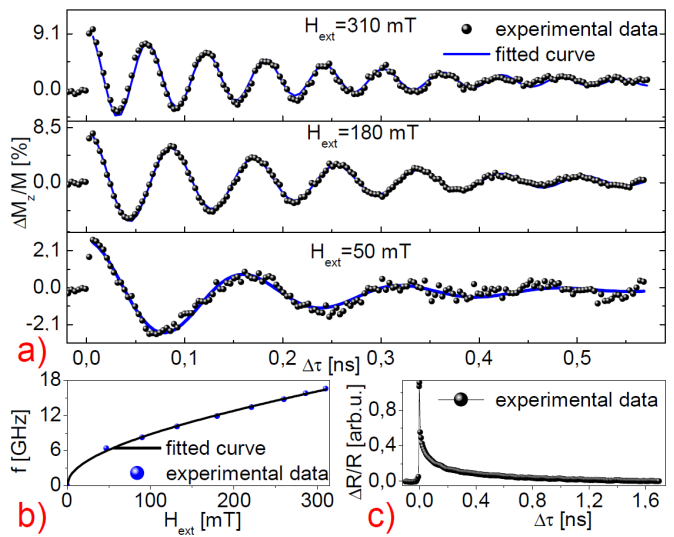

Fig. 2. The results for $\mathrm{Fe}(0.5 \mathrm{~nm}) / \mathrm{Au}(0.95 \mathrm{~nm})(111)$ film. (a) Magnetization precession induced by ultrafast pulses recorded in $H_{\text {ext }}$ with corresponding fitted lines (see text). (b) Precession frequency dependence on $H_{\text {ext }}$. (c) Pump-induced change of sample reflectivity.

gle channel of balanced detector (BD) on $\mathrm{Fe}(0.5 \mathrm{~nm}) /$ $\mathrm{Au}(0.95 \mathrm{~nm})$ multilayer structure is presented. Signal quality of $\Delta R / R$ well defines the zero scale of ODL and evidences good overlapping of spots on the whole long translation stage distance.

A special method of autocompensation of stage motion misalignments [4] was used which guarantees precise overlapping of two laser spots over the long distance without applying additional equipment which could cause temporal laser pulse broadening [5]. Pump and probe beams were directed on the sample under angle $\approx 7^{\circ}$ and $\approx 33^{\circ}$, respectively, relative to the normal of sample plane. The probe beam after reflection from the sample (S1) placed inside electromagnet (EM) and passing through the Wollaston prism is detected by amplified balanced Si detector BD. The differential channel output of $\mathrm{BD}$ was fed the input of the first phase-sensitive detector synchronized with PEM, and the output was directed to the input of second lock-in amplifier, whose reference was connected to chopper controller. Sufficient DAQ card timing parameters assure processing of differential signal and ODL location in fast real-time mode.

Experimental arrangement for measurements MSHG on transparent magnetic medium is shown in Fig. 1b. Laser beam with vertical $X$ direction polarization is directed onto the sample (S2) through a series of optical elements. Double achromatic lenses (L3, L4, L5) were used for focusing and forming incident light in a spots diameter of approximately $30 \mu \mathrm{m}$ on the sample, and $20 \mu \mathrm{m}$ on the detector, respectively. The bandpass filter (BF) blocks photons with a wavelength different than $400 \mathrm{~nm}$. Precise Rochon prism was used as the analyzer (Pol). The ultra-low noise photon counting system (PHC) determines high timing resolution and very low dark count rate (single-photon counting module id100-20 from id-Quantique with $20 \mu \mathrm{m}$ photosensitive area). In the experiment the sample plane $x y$ is perpendicular to plane of incidence of light beam and the sample is placed inside of electromagnet (EM) with applied magnetic field up to $2 \mathrm{kOe}$ along the $Y$ direction. The setup was used for investigating MSHG rotational optical anisotropy in magnetic garnet films. The angle of the sample rotation was controlled by motorized rotation stage and the temperature dependent measurements were possible with homemade heater stage. The subsetup shown in Fig. 1b allows also for simultaneous measurement of linear longitudinal Kerr effect based on polarization modulation method. Hight power laser diode (LD) for $\lambda=405 \mathrm{~nm}$ is used as a light source. LD beam with $s$-polarization is focused at angle of $\approx 22^{\circ}$ on the sample. Reflected beam is directed through the PEM and analyzer on the photodiode (D).

\section{Results and discussion}

In picosecond time scale evolution of the magnetization $M$ is well described by classical Landau-LifshitzGilbert (LLG) equation [1]. We studied magnetization dynamics in sputter deposited $\mathrm{Fe}(0.5 \mathrm{~nm}) / \mathrm{Au}(0.95 \mathrm{~nm})$ (111) multilayer on the GaAs (001) substrate, with easy-plane magnetization. TRMOKE signal measurements were done as a function of applied external magnetic field $\boldsymbol{H}_{\text {ext }}$. The results of relative change of magnetization versus delay time $t=\Delta \tau$ are shown in Fig. 2a for selected $H_{\text {ext }}$ values. The experimental data were fitted 
with the formula: $\Delta M_{z}(t) / M_{z}=A \sin (2 \pi f t+\psi) \mathrm{e}^{-\frac{t}{\tau_{\mathrm{LLG}}}}$, where fitting parameters $A, f$ and $\psi$ are the amplitude, frequency and phase of precession, respectively. The $\tau_{\text {LLG }}$ is the relaxation time of magnetization vector and is related to the Gilbert damping parameter with following relationship [6]: $\alpha=2\left(\gamma \tau_{\mathrm{LLG}}\left(2 H_{x}+H_{\mathrm{az}}\right)\right)^{-1}$ where $\gamma$ is gyromagnetic factor, $H_{\mathrm{az}}$ is effective anisotropy field and $H_{x}=H_{\text {ext }} \cos \phi$ is a component of $\boldsymbol{H}_{\text {ext }}$ in the sample plane. $\boldsymbol{H}_{\text {ext }}$ was directed at an angle $\phi \approx$ $10^{\circ}$ relative to the sample plane. As it is shown in Fig. 2a, amplitude and frequency of the precession increases with $H_{\text {ext }}$. The frequencies $f$ as a function of $H_{\text {ext }}$ change from 6 to $16 \mathrm{GHz}$ as is shown in Fig. $2 \mathrm{~b}$. Adapted to our experimental geometry the Kittel formula is: $f=\frac{g \gamma \mu_{0}}{4 \pi} \sqrt{H_{x}\left(H_{x}+H_{\mathrm{az}}\right)}$, where $\mu_{0}$ is vacuum magnetic permeability and $g=2.089$ is Fe Landé factor [7]. The data in Fig. $2 \mathrm{~b}$ were fitted with the above formula with $H_{\mathrm{az}}=1.075 \pm 0.188 \mathrm{~T}$. With this value, the calculated $\alpha$ factor decreases slightly with $H_{\text {ext }}$. The average $\alpha$ value is $\alpha=0.035 \pm 0.009$.

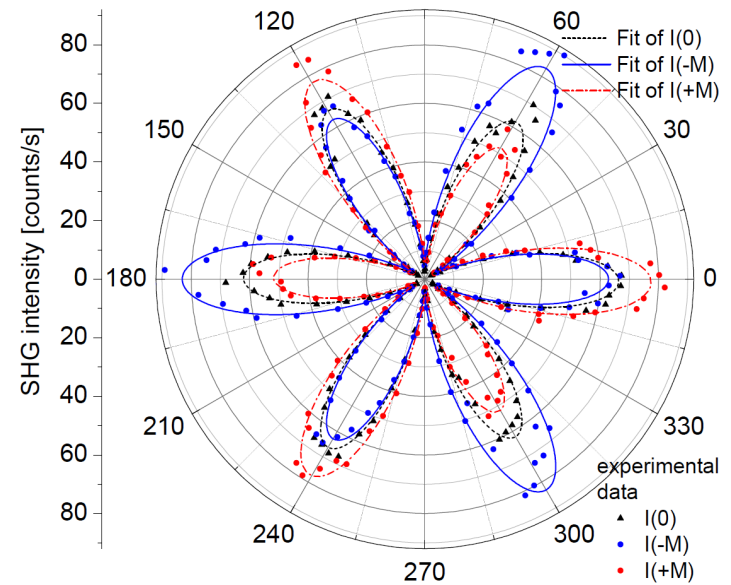

Fig. 3. Rotational anisotropy of the SHG intensity for (111) YIG garnet film for $X X$ polarizations combination. Lines denote the best fits for $I_{X X}(2 \omega, \varphi)$ (see text).

The study of MSHG was performed on $0.5 \mu \mathrm{m}$ thick epitaxial YIG garnet film grown on a (111) oriented gadolinium gallium garnet (GGG) substrate. The intensity of $2 \omega$ light coming from the surface of the film was recorded as a function of azimuthal angle $\varphi$ of sample rotation. Combination of $X$ input and $X$ output polarization was used. For $\boldsymbol{H}_{\text {ext }}=0$ the sixfold symmetry of rotational anisotropy is seen in Fig. 3, in agreement with (111) film plane symmetry. For nonzero $\boldsymbol{H}_{\text {ext }}$ the symmetry reduces to threefold according to the formula [8]: $I_{X X}(2 \omega, \varphi)=A \cos ^{2} 3 \varphi+B M^{2}+2 C M \cos 3 \varphi$. Considerable change of MSHG signal with $\boldsymbol{H}_{\text {ext }}$ sign is seen (Fig. 3) in correspondence to sign change in magnetization $\boldsymbol{M}$. The solid, dashed and dash-dotted lines representing best fitting to above formula describe well the experimental data. The experimental setup allows also to measure hysteresis loops of MSHG signal. Analysis of the results is beyond of scope of present paper.

\section{Conclusions}

We present experimental setup and successfully applied it to study of ultrafast magnetization dynamics processes in $\mathrm{Fe} / \mathrm{Au}$ multilayers structures. From the experimental data obtained the effective anisotropy field and Gilbert damping parameter were determined for the investigated structure. In the second experiment MSHG were measured for thin garnet YIG films. Measured rotational anisotropy allowed us to determine both crystallographic symmetry of the film studied and significant change of it with applied magnetic field.

\section{References}

[1] A. Kirilyuk, A.V. Kimel, Th. Rasing, Rev. Mod. Phys. 82, 2731 (2010) and references therein.

[2] B. Koopmans, M. Van Kampen, W.J.M. De Jonge, J. Phys.: Condens. Matter 15, S723 (2003).

[3] B. Koopmans, M. van Kampen, J.T. Kohlhepp, W.J.M. de Jonge, J. Appl. Phys. 87, 5070 (2000).

[4] J. Stenholm Teppo, T. Soini Juhani, E. Hänninen Pekka, Measur. Sci. Technol. 15, 2001 (2004).

[5] S. Capinski William, J. Maris Humphrey, Rev. Sci. Instrum. 67, 2720 (1996).

[6] M. Van Kampen, C. Jozsa, J.T. Kohlhepp, P. LeClair, L. Lagae, W.J.M. De Jonge, B. Koopmans, Phys. Rev. Lett. 88, 227201 (2002).

[7] Z. Frait, Czechoslovak J. Phys. 27, 185 (1977).

[8] V.V. Pavlov, R.V. Pisarev, A. Kirilyuk, Th. Rasing, Phys. Rev. Lett. 78, 2004 (1997). 